\title{
Barriers to the Adoption of Electronic Medical Record System in Ethiopia: A Systematic Review
}

\author{
Delelegn Emwodew \\ Yehualashet (iD) \\ Binyam Tariku Seboka (iD I \\ Getanew Aschalew Tesfa (D) \\ Abel Desalegn Demeke ${ }^{2}$ \\ Endris Seid Amede ${ }^{3}$ \\ 'Department of Health Informatics, Dilla \\ University, Dilla, Gedeo, Ethiopia; \\ ${ }^{2}$ Department of Nursing, Dilla University, \\ Dilla, Gedeo, Ethiopia; ${ }^{3}$ Department of \\ Psychiatry, Dilla University, Dilla, Gedeo, \\ Ethiopia
}

Background: Electronic medical records (EMRs) can improve the quality of health care and patient safety. Various countries have gone through the local application of EMRs to various health care organizations in national implementation and integration of EMRs. Ethiopia lags far in the back in this regard, as solely some hospitals have implemented EMR.

Objective: This study aimed to identify barriers to the adoption of EMRs in Ethiopia through a systematic review of the literature.

Methods: PubMed, Semantic Scholar, and Google Scholar have been searched for applicable articles. The search method focuses on peer-reviewed, empirical research conducted in Ethiopia. The ultimate set that met the inclusion standards was 9 studies. The authors extracted, analyzed, and summarized empirical results associated with EMR barriers in these studies.

Results: This systematic review identified the following 17 barriers to EMR adoption: absence of EMR training, limited access to computers, insufficient computer literacy, deficiency of EMR knowledge, inadequate technical help, absence of EMR manual, negative attitude to EMR, limited internet access, lack of management support, electric power interruption, absence of perceived system quality, absence of perceived information quality, lack of willingness, the complexity of the system, performance expectancy, effort expectancy, and lack of IT qualification.

Conclusion: The most common barriers for EMR adoption are absence of EMR training, limited access to a computer, poor computer literacy, poor EMR knowledge, lack of technical support, and absence of an EMR manual. As this study summarizes the available evidence regarding barriers to adopting EMR in Ethiopia, future research will rest on this evidence and specialize in building a proper framework for EMR implementation in Ethiopia.

Keywords: electronic medical record, electronic health record, barriers, adoption, Ethiopia, systematic review

\section{Introduction}

Electronic Medical Records (EMRs) is a computerized electronic file system used to capture, store, and share information between health care providers in an organization, which supports the delivery of patient health services. ${ }^{1}$ It helps to enhance healthcare quality through enhancing workflow, lowering medical malpractice, minimizing price and therapy time, increasing revenue, improving patient care through creating a stronger linkage to all caregivers, lowering the want for file hose and people for the retrieval and filing of medical records. ${ }^{2,3}$

Despite the high expectations and activity in EMR as a principal pressure to enhance the standard, quality, continuity, safety, and effectiveness of healthcare worldwide, the adoption rate is incredibly low. ${ }^{4-6}$ Over $50 \%$ of EMRs may fail or fail to be used effectively within the world. ${ }^{5,7}$
Correspondence: Delelegn Emwodew Yehualashet

Department of Health Informatics, Dilla

University, Dilla, Gedeo, Ethiopia

$\mathrm{Tel}+251976061907$

Email delelegn1244@gmail.com 
Over the previous few decades, many countries are dedicated themselves to the countrywide implementation of EMRs to boost healthcare systems and better control human health needs. ${ }^{8}$ As in many countries, the Ethiopian Ministry of Health has acknowledged the importance of EMRs for quality enhancement in healthcare delivery. In 2013, the Ethiopian Ministry of Health adopted the EMR and planned to extend it to succeed in all hospitals, however, few health facilities have implemented EMRs. ${ }^{9}$

Given the massive quantity of resources supplied for the implementation of EMR, figuring out barriers to the adoption of EMR is vital to its effective implementation. Although researches are being carried out to recognize the barriers to the adoption of EMRs in Ethiopia, there has been no systematic review of those studies. Thus, our study aimed to identify barriers to the adoption of EMRs in Ethiopia through a systematic review of the literature. The findings of this study will aid policymakers in planning and developing strategies to extend EMR adoption.

\section{Methods}

A systematic literature review was carried out based on the Preferred Reporting Items for Systematic reviews and Meta-Analyses (PRISMA). ${ }^{10}$ PRISMA was used to show the flow of the study at each stage of the review process. The PRISMA flow diagram is also used to map the number of records identified, included, and excluded and the reasons for exclusion.

\section{Source of Information and Search Strategy}

PubMed, Semantic Scholar, and Google Scholar were searched from 2 to 15 March 2021. To amplify the possibilities of finding all researches carried out in Ethiopia, two common search terms, separated by using operator "OR", were used: "electronic medical record" AND "Ethiopia" OR "electronic health record" AND "Ethiopia".

\section{Eligibility Criteria}

The inclusion and exclusion criteria's were: 1) articles focusing solely on EMR or EHR, but no longer other electronic systems used in healthcare (for instance on IT systems, or Personal Health Records (PHRs)), 2) articles assessing barriers to the implementation and/or adoption of EMR/EHR, 3) articles published in scientific journals-conference articles and unpublished work were excluded, 4) articles primarily based on empirical studies, and 5) articles where the country of data collection was in Ethiopia.

\section{Selection Process}

Initially, two independent reviewers screened all titles and abstracts for probably eligible articles. Articles that failed to meet the inclusion criteria were removed from the list. Discrepancies about exclusions between the two reviewers were resolved through discussion. Finally, all reviewers assessed the full text of each article individually to create the ultimate selection of important studies to include.

\section{Data Extraction and Analysis}

Articles that met the eligibility criteria were further analyzed and therefore the following items had been extracted from each article: research methods, data collection methods, sample size, and sampling techniques. Finally, the empirical results associated with EMR adoption barriers were extracted from each article.

Meta-analysis of results was not attempted because of variations between studies in terms of research approaches and type of participants. For instance, research ${ }^{11}$ used a qualitative method to understand the hindering factors for EMR adoption, while the remaining studies used quantitative methods. Statistical interpretation supported the findings of ${ }^{11}$ was not feasible, so meta-analysis was not possible. But, the analytical method employed by previous systematic reviews ${ }^{12,13}$ were used in the current study. In this manner, the barriers were analyzed based on the rate of existence within the studies. This method can yield trustworthy results in our study.

\section{Results}

\section{Study Selection}

The selection process and the associated inclusion criteria are presented in Figure 1. A total of 1264 studies had been recognized for the first screening. Then, 684 studies have been removed after being marked as duplicates. After being managed for duplicates, the titles and abstracts of 580 studies have been examined. Subsequently, 83 studies had been placed for a full-text review. A total of 74 studies have been excluded based on the full-text assessment. Finally, 9 studies were included in the review.

\section{Study Characteristics}

Included articles varied across some areas, as shown in Table 1. Six studies used a quantitative research method, two 


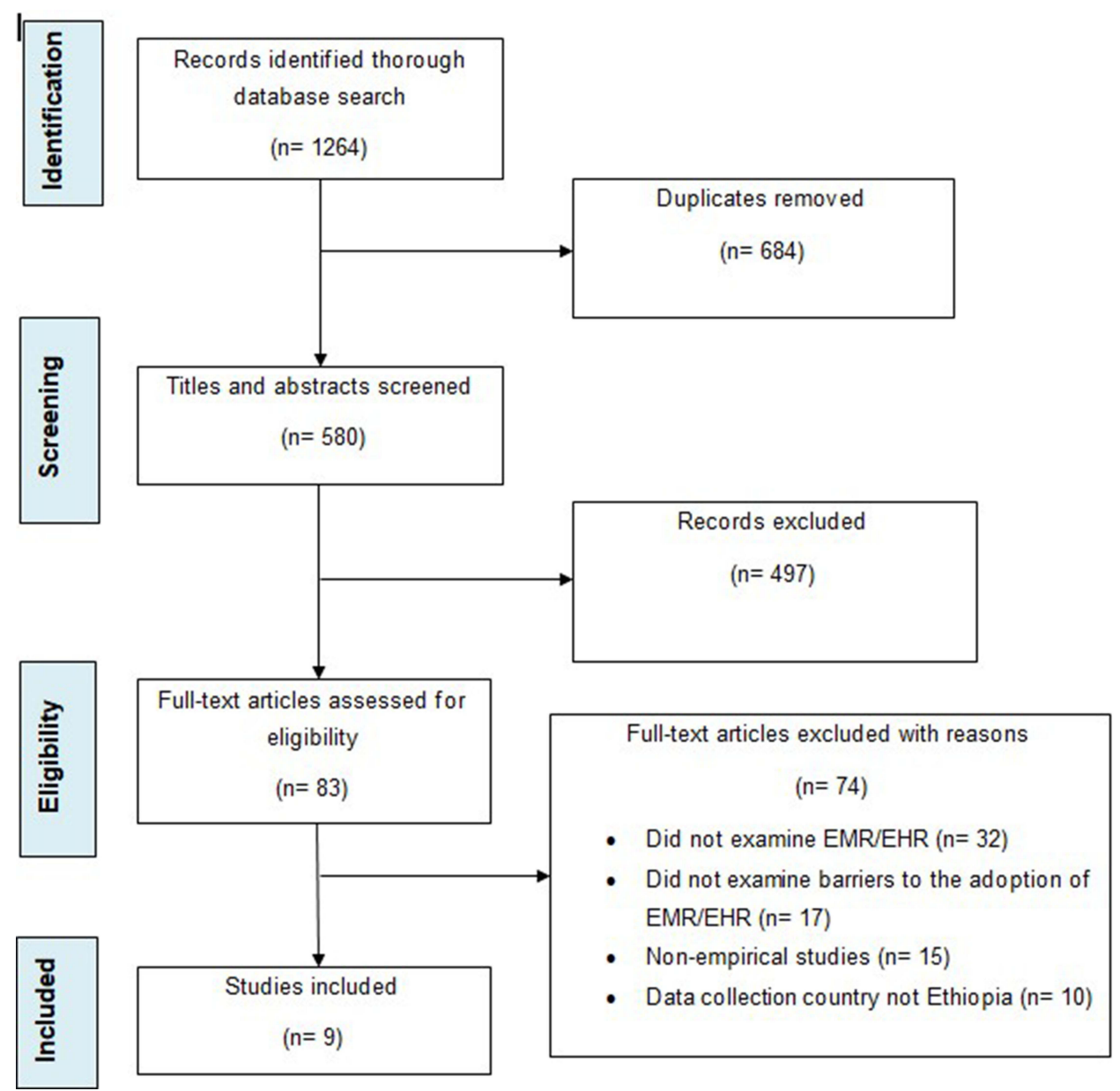

Figure I PRISMA flowchart showing the selection process and the associated inclusion criteria.

Notes: PRISMA adapted from Liberati A, Altman D, Tetzlaff J, et al. The PRISMA statement for reporting systematic reviews and meta-analyses of studies that evaluate health care interventions: explanation and elaboration. Journal of Clinical Epidemiology. 2009;62(10)el-e34. Creative Commons. ${ }^{42}$

Abbreviations: PRISMA, preferred reporting items for systematic reviews and meta-analysis; EMR, electronic medical record; EHR, electronic health record.

using a mixed approach and one remaining using a qualitative approach. All of these studies were conducted in hospital areas, except this one, which was conducted in hospital and primary care. ${ }^{14}$ Different types of respondents have been included in the studies. All studies involved a mix of different health professionals such as laboratory staff, pharmacists, midwives, and others, ${ }^{14}$ doctors, nurses, pharmacists, and health management information system (HMIS) staff. ${ }^{9}$

Table I Characteristics of Included Studies

\begin{tabular}{|c|c|c|c|}
\hline Reference/ Year of Publication & Type of Research & Method of Data Collection & Number of Participants/ Sampling Strategy \\
\hline Awol et $\mathrm{al}^{36} / 2020$ & Mixed & Questionnaire \& interview & 45I/ Random sampling \\
\hline Biruk et $\mathrm{al}^{37} / 2014$ & Quantitative & Questionnaire & 606/ Random sampling \\
\hline Ahmed et $\mathrm{al}^{38} / 2020$ & Quantitative & Questionnaire & 420/ Random sampling \\
\hline Berihun et $\mathrm{al}^{14 / 2} 2020$ & Quantitative & Questionnaire & 616/ Random sampling \\
\hline Yehualashet et $\mathrm{al}^{39} / 2015$ & Quantitative & Questionnaire & 428/ Random sampling \\
\hline Tilahun and Fritz ${ }^{9} / 2015$ & Quantitative & Questionnaire & 406/ Random sampling \\
\hline Tilahun and Fritz ${ }^{40} / 2015$ & Quantitative & Questionnaire & 332/ Random sampling \\
\hline Gebre-Mariam et al ${ }^{11 /} 2012$ & Qualitative & Questionnaire \& interview & 30/ Convenience sampling \\
\hline Berhe et $\mathrm{al}^{41} / 2017$ & Mixed & Questionnaire \& FGD & 255/ Sampling strategy not provided \\
\hline
\end{tabular}


Table 2 Barriers to the Adoption of EMR in Ethiopia and Frequency of Occurrences

\begin{tabular}{|c|c|c|c|c|}
\hline No. & Barriers & References & Frequency & $\%$ \\
\hline I & Lack of EMR training & {$[9,11-37,39,40]$} & 7 & 77.8 \\
\hline 2 & Limited computer access & {$[9,36,37,39,40]$} & 5 & 55.6 \\
\hline 3 & Lack of computer literacy & {$[|4,36,37,39,4|]$} & 5 & 55.6 \\
\hline 4 & Lack of knowledge on EMR & {$[14,36,37]$} & 3 & 33.3 \\
\hline 5 & Lack of technical support & {$[11,38,40]$} & 3 & 33.3 \\
\hline 6 & Absence of EMR manual & {$[14,39,40]$} & 3 & 33.3 \\
\hline 7 & Negative attitude towards EMR & {$[36,37]$} & 2 & 22.2 \\
\hline 8 & Limited internet access & {$[36,4 I]$} & 2 & 22.2 \\
\hline 9 & Lack of management support & {$[14,39]$} & 2 & 22.2 \\
\hline 10 & Electric power interruption & {$[39,4 I]$} & 2 & 22.2 \\
\hline 11 & Lack of perceived system quality & {$[9,40]$} & 2 & 22.2 \\
\hline 12 & Lack of perceived information quality & {$[9,40]$} & 2 & 22.2 \\
\hline 13 & Lack of willingness & [37] & 1 & II.I \\
\hline 14 & Complexity of the system & [37] & 1 & II.I \\
\hline 15 & Performance expectancy & [38] & 1 & II.I \\
\hline 16 & Effort expectancy & [38] & 1 & II.I \\
\hline 17 & Lack of IT qualification & [9] & I & II.I \\
\hline
\end{tabular}

\section{Barriers to EMR Adoption in Ethiopia}

A total of 17 barriers have been identified across the 9 studies as presented in Table 2. The identified barriers are lack of EMR training, limited access to computers at work, lack of computer literacy, lack of EMR knowledge, lack of technical support, lack of EMR manual, health professionals negative attitude towards EMR, limited internet access in the workplace, lack of management support, electric power interruption, lack of perceived system quality, lack of perceived information quality, lack of willingness, the complexity of the system, performance expectancy, effort expectancy, and lack of information technology (IT) qualification.

The 17 barriers are organized by the frequency of occurrences among the studies, with the most frequently listed first. The frequency rates of the 17 barriers are: the "Lack of EMR training" appeared in seven of the nine studies (77.8\%), "Limited access of computer" and "Lack of computer literacy" appeared in five of the nine studies (55.6\%); "Lack of knowledge", "Lack of technical support", and "absence of EMR manual", each appeared in three of the nine studies (33.3\%); "Negative attitude towards EMR", "Limited internet access", "Lack of management support", "Electric power interruption", "Lack of perceived system quality" and "Lack of perceived information quality" each appeared in two of the nine studies (22.2\%); five barriers, namely: "Lack of willingness to implement the system", "Complexity of the system",
"Performance expectancy", "Effort expectancy", and "Lack of IT qualification" each appeared once in the nine articles (11.1\%).

\section{Discussion}

Our systematic review found a variety of potential barriers to the adoption of EMR systems in Ethiopia. The most common barriers to EMR adoption are Lack of EMR training, limited access to computers, lack of computer literacy, lack of EMR knowledge, lack of technical support, and lack of EMR manuals.

Lack of EMR training is often declared as an obstacle. A systematic review of the perceptions of health care professionals about EMR adoption in the Gulf Cooperation Council countries has provided a lack of training as a major barrier to EMR adoption. ${ }^{15}$ Similarly, evidence from western Canada states that the lack of proper practical training and support for EMR systems has affected the success of EMR implementation. ${ }^{16}$ In another study from India, two-thirds of medical professionals cited a lack of EMR training as a barrier to EMR adoption. ${ }^{17}$ Accordingly, training should be considered as an important determinant for the successful implementation of EMR systems.

One of the most commonly reported barriers is the lack of a computer in the office. Similarly, a study by Norwegian physicians on the use of EMR systems reported that some of the computers and other hardware 
required to run EMR properly were not present in some practices and that this problem prevented EMR from being widely adopted. ${ }^{18}$ Implementing EMR systems requires considerable hardware, including computers, cable, and Internet connections. Therefore, the successful implementation of EMR by healthcare organizations should take into account the allocation of appropriate technical resources and a percentage of total revenue.

The limited capacity of health professionals in computer literacy is also a persistent obstacle. The study was supported by a study conducted in Malaysia, ${ }^{19}$ Taiwan, ${ }^{20}$ and Iran. $^{21}$ Consistently, several systematic reviews ${ }^{22-25}$ suggest that a lack of computer literacy is the most common barrier to EMR adoption. Three studies conducted in Saudi Arabia report that computer literacy is closely related to health care professionals' EHR acceptance, ${ }^{26}$ health care professionals' EHR use, ${ }^{27}$ and health professionals' satisfaction with HER. ${ }^{28}$ Furthermore, studies conducted in developing countries have shown that poor computer skills of health professionals are related to poor implementation of EMR systems. ${ }^{29-31}$

The present study also found that lack of EMR knowledge is a major barrier to EMR adoption. Similarly, a study conducted in Iran on nurse readiness to implement EMR found that lack of knowledge was a major obstacle to the success of EMR implementation. ${ }^{32}$ A similar study in northern Ghana found that EHR knowledge was an important determinant of healthcare provider's readiness to adopt EHRs. ${ }^{33}$ Another important issue is the negative attitude of health care professionals towards the EMR system. This exploration is consistent with a systematic review of the perceptions of health care professionals on EHR adoption in the Gulf Cooperation Council countries, in which negative perception is a major challenge to adopt an EHR. ${ }^{15}$ This is a good explanation for the need for professionals to be aware of the EMR system before implementing it so that they have a good attitude and develop their readiness for better adaptation of the system.

Lack of management support has also been identified as a major problem hindering the implementation of EMR systems. Whether managers support EMR adoption and trust the benefits of EMR will have an impact on clinicians' level of EMR adoption. ${ }^{6,34,35}$ Therefore, managers at all levels of the health care system must be committed to supporting EMR implementation.

Overall, this study summarizes the results of current empirical studies and identifies barriers to the widespread adoption of the EMR system in Ethiopia. To promote health care delivery and patient care quality, managers and other stakeholders in healthcare organizations need to be aware of these barriers that hinder EMR implementation. There are some important limitations to this study. Although the authors did a thorough search, only a limited number of articles $(n=9)$ were found. This may be due to limited research on e-health in Ethiopia.

\section{Conclusion}

In this paper, barriers to the adoption of the EMR system in Ethiopia are identified through a systematic review of the literature. Lack of EMR training, limited access to computers, health professional's limited computer literacy, deficiency of EMR knowledge, absence of technical support, and absence of EMR manual were the most common barriers to the adoption of EMR. This knowledge update about EMR barriers is important for policymakers, health informatics professionals, academics, clinicians, and EMR vendors. EMR policymakers and implementers, such as hospital managers and project leaders, can use this information to understand what barriers exist in their specific situation to identify appropriate interventions to address these barriers. From a practical point of view, policymakers can use the results of this study to plan and design policies to increase EMR adoption. Additionally, EMR vendors can use the findings in system development and marketing. Researchers can also use this information to investigate reported barriers in various settings and regions (eg investigating type and frequency of technical issues with EMR systems). As this study summarizes the available evidence regarding barriers to adopting EMR in Ethiopia, future studies will rest on this evidence and specialize in building an appropriate framework for EMR adoption in Ethiopia.

\section{Author Contributions}

All authors made a significant contribution to the work reported, whether that is in the conception, study design, execution, acquisition of data, analysis, and interpretation, or in all these areas; took part in drafting, revising, or critically reviewing the article; gave final approval of the version to be published; have agreed on the journal to which the article has been submitted; and agree to be accountable for all aspects of the work.

\section{Disclosure}

The authors report no conflicts of interest in this work. 


\section{References}

1. Hamilton C. The WHO-ITU national eHealth strategy toolkit as an effective approach to national strategy development and implementation. Stud Health Technol Inform. 2013;192:913-916.

2. Bagayoko CO, Anne A, Fieschi M, Geissbuhler A. Can ICTs contribute to the efficiency and provide equitable access to the health care system in Sub-Saharan Africa? The Mali experience. Yearb Med Inform. 2011;6(1):33-38.

3. Williams F, Boren SA. The role of the electronic medical record (EMR) in care delivery development in developing countries: a systematic review. Inform Prim Care. 2008;16(2):139-145.

4. Terry AL, Brown JB, Bestard Denomme L, Thind A, Stewart M. Perspectives on electronic medical record implementation after two years of use in primary health care practice. J Am Board Fam Med. 2012;25(4):522-527. doi:10.3122/jabfm.2012.04.110089

5 . Willyard C. Focus on electronic health records: electronic records pose dilemma in developing countries. Nat Med. 2010;16(3):249. doi:10.1038/nm0310-249a

6. Miller RH, Sim I. Physicians? use of electronic medical records: barriers and solutions. Health Aff. 2004;23(2):116-126. doi:10.1377/ hlthaff.23.2.116

7. Ketikidis P, Dimitrovski T, Lazuras L, Bath PA. Acceptance of health information technology in health professionals: an application of the revised technology acceptance model. Health Informatics $J$. 2012;18 (2):124-134. doi:10.1177/1460458211435425

8. AlJarullah A, El-Masri S. A novel system architecture for the national integration of electronic health records: a semi-centralized approach. J Med Syst. 2013;37(4):1-20. doi:10.1007/s10916-0139953-4

9. Tilahun B, Fritz F. Comprehensive evaluation of EMR system use and user satisfaction at five low-resource setting hospitals in Ethiopia. JMIR Med Inform. 2015;3(2):e22. doi:10.2196/medinform.4106

10. Moher D, Liberati A, Tetzlaff J, Altman DG. Preferred reporting items for systematic reviews and meta-analyses: the PRISMA statement. PLoS Med. 2009;6(7):e1000097. [PubMed PMID: 19621072]. doi:10.1371/journal.pmed.1000097

11. Gebre-Mariam M, Borycki E, Kushniruk A, Purkis M An Electronic Medical Record (EMR) implementation framework for HIV care and treatment facilities in Ethiopia. 2012;11.

12. Khan SU, Niazi M, Ahmad R. Barriers in the selection of offshore software development outsourcing vendors: an exploratory study using a systematic literature review. Inf Softw Tech. 2011;53 (7):693-706. doi:10.1016/j.infsof.2010.08.003

13. Kruse CS, Goetz K. Summary and frequency of barriers to adoption of CPOE in the U.S. J Med Syst. 2015;39(2):15. doi:10.1007/s10916015-0198-2

14. Berihun B, Atnafu DD, Sitotaw G. Willingness to use Electronic Medical Record (EMR) system in healthcare facilities of Bahir Dar City, Northwest Ethiopia. Biomed Res Int. 2020;2020:3827328. doi: $10.1155 / 2020 / 3827328$

15. Alanazi B, Butler-Henderson K, Alanazi M. Perceptions of healthcare professionals about the adoption and use of EHR in Gulf cooperation council countries: a systematic review. BMJ Health Care Inform. 2020;27(1):e100099. doi:10.1136/bmjhci-2019-100099

16. Ludwick DA, Doucette J. Primary care physicians' experience with electronic medical records: barriers to implementation in a fee-forservice environment. Int J Telemed Appl. 2009;853524. doi: 10.1155/ 2009/853524

17. Loomis GA, Ries S, Saywell RM, Thakker NR. If electronic medical records are so great, why aren't family physicians using them? J Fam Pract. 2002;51(7):636-641.

18. Laerum H, Ellingsen G, Faxvaag A. Doctors' use of electronic medical records systems in hospitals: cross sectional survey. $\mathrm{Br}$ Med J. 2001;323(7325):1344-1348. doi:10.1136/bmj.323.7325.1344
19. Mukred M, Yusof ZM, Alotaibi FM, Mokhtar UMIA, Fauzi F. The key factors in adopting an electronic records management system (ERMS) in the educational sector: a UTAUT-based framework. IEEE Access. 2019;7:35963-35980. doi:10.1109/ACCESS.2019.2904617

20. Huang W-M, Chen T, Hsieh C-W An empirical study on the physicians' behavioral intention with electronic medical record systems in Taiwan. PACIS; 2014:160.

21. Gagnon M, Kebir E, Kengne P, et al. Electronic health record acceptance by physicians: testing an integrated theoretical model. $J$ Biomed Inform. 2014;48:17-27. doi:10.1016/j.jbi.2013.10.010

22. Gagnon MP, Desmartis M, Labrecque M, et al. Systematic review of factors influencing the adoption of information and communication technologies by healthcare professionals. J Med Syst. 2012;36 (1):241-277. doi:10.1007/s10916-010-9473-4

23. Li J, Talaei-Khoei A, Seale H, Ray P, Macintyre CR. Health care provider adoption of eHealth: systematic literature review. Interact J Med Res. 2013;2(1):e7. doi:10.2196/ijmr.2468

24. McGinn CA, Grenier S, Duplantie J, et al. Comparison of user groups' perspectives of barriers and facilitators to implementing electronic health records: a systematic review. BMC Med. 2011;9 (1):1-10. doi:10.1186/1741-7015-9-46

25. Najaftorkaman M, Ghapanchi AH, Talaei-Khoei A, Ray P. A taxonomy of antecedents to user adoption of health information systems: a synthesis of thirty years of research. J Assoc Inf Sci Technol. 2015;66(3):576. doi:10.1002/asi.23181

26. Hasanain RA, Vallmuur K, Clark M. Electronic medical record systems in Saudi Arabia!: knowledge and preferences of healthcare professionals. J Health Inform Dev Ctries. 2015;9(1):23-31.

27. Nour El Din MM. Physicians' use of and attitudes toward electronic medical record system implemented at a teaching hospital in Saudi Arabia. J Egypt Public Health Assoc. 2007;82(5-6):347-364.

28. Alasmary M, El Metwally A, Househ M. The association between computer literacy and training on clinical productivity and user satisfaction in using the electronic medical record in Saudi Arabia. J Med Syst. 2014;38(8):1-13. doi:10.1007/s10916-014-0069-2

29. Kgasi MR, Kalema BM. Assessment E-health readiness for rural South African areas. $J$ Ind Intell Inf. 2014;2(2):131-135. doi:10.12720/jiii.2.2.131-135

30. Yogeswaran P, Wright G. EHR implementation in South Africa: how do we get it right? Stud Health Technol Inform. 2010;160(Pt 1):396-400.

31. Fraser HS, Biondich P, Moodley D, Choi S, Mamlin BW, Szolovits P. Implementing electronic medical record systems in developing countries. Inform Prim Care. 2005;13(2):83-95.

32. Mahdi H-K, Reza S, Hamid B. Nurses readiness and electronic health records. Acta Inform Med. 2015;23(2):105. doi:10.5455/aim.20 15.23.105-107

33. Adjorlolo S, Ellingsen G. Readiness assessment for implementation of electronic patient record in Ghana: a case of university of Ghana hospital. J Health Inform Dev Ctries. 2013;7(2):128-140.

34. Vishwanath A, Scamurra SD. Barriers to the adoption of electronic health records: using concept mapping to develop a comprehensive empirical model. Health Informatics J. 2007;13(2):119-134. doi: $10.1177 / 1460458207076468$

35. Reardon JL, Davidson E. An organisational learning perspective on the assimilation of electronic medical records among small physician practices. Eur J Inf Syst. 2007;16(6):681-694. doi:10.1057/palgrave. ejis.3000714

36. Awol SM, Birhanu AY, Mekonnen ZA, et al. Health professionals' readiness and its associated factors to implement electronic medical record system in four selected primary hospitals in Ethiopia. Adv Med Educ Pract. 2020;11:147-154. doi:10.2147/AMEP.S233368

37. Biruk S, Yilma T, Andualem M, Tilahun B. Health Professionals “ readiness to implement electronic medical record system at three hospitals in Ethiopia: a cross-sectional study. BMC Med Inform Decis Mak. 2014;14(1):115. doi:10.1186/s12911-014-0115-5 
38. Ahmed MH, Bogale AD, Tilahun B, et al. Intention to use electronic medical record and its predictors among health care providers at referral hospitals, north-West Ethiopia, 2019: using unified theory of acceptance and use technology 2(UTAUT2) model. BMC Med Inform Decis Mak. 2020;20(1):207.

39. Yehualashet G, Asemahagn M, Tilahun B. The attitude towards and use of electronic medical record system by health professionals at a referral hospital in Northern Ethiopia: a cross-sectional study. J Health Inform Afr. 2015;3(1):19-29.

40. Tilahun B, Fritz F. Modeling antecedents of electronic medical record system implementation success in low resource setting hospitals. BMC Med Inform Decis Mak. 2015;15(1):61. doi:10.1186/s12911015-0192-0
41. Berhe M, Gebremariam K, Gebregergs GB, Gebrehiwot T. Evaluation of electronic medical record implementation from user's perspectives in Ayder referral hospital Ethiopia. $J$ Health Med Inform. 2017;8(1):2. doi:10.4172/2157-7420.1000249

42. Liberati A, Altman D, Tetzlaff J, et al. The PRISMA statement for reporting systematic reviews and meta-analyses of studies that evaluate health care interventions: explanation and elaboration. ournal of Clinical Epidemiology. 2009;62(10)e1-e34.

\section{Publish your work in this journal}

The Journal of Multidisciplinary Healthcare is an international, peerreviewed open-access journal that aims to represent and publish research in healthcare areas delivered by practitioners of different disciplines. This includes studies and reviews conducted by multidisciplinary teams as well as research which evaluates the results or conduct of such teams or healthcare processes in general. The journal covers a very wide range of areas and welcomes submissions from practitioners at all levels, from all over the world. The manuscript management system is completely online and includes a very quick and fair peer-review system. Visit http://www.dovepress.com/testimonials. php to read real quotes from published authors. 